\title{
CURRENT CHALLENGES AND SOLUTION APPROACHES IN EMPIRICAL ENGINEERING DESIGN RESEARCH - A WORKSHOP FOR EMPIRICAL RESEARCH
}

\author{
S. Üreten ${ }^{1, \bigotimes}$, M. Eisenmann ${ }^{2}$, T. Nelius ${ }^{2}$, E. Garrelts ${ }^{3}$, D. Krause ${ }^{1}$ and \\ S. Matthiesen ${ }^{2}$ \\ ${ }^{1}$ Hamburg University of Technology, Germany, ${ }^{2}$ Karlsruhe Institute of Technology, Germany, ${ }^{3}$ University of \\ Stuttgart, Germany \\ $\triangle$ selin.uereten@tuhh.de
}

\begin{abstract}
The requirements on validity for studies in design research are very high. Therefore, this paper aims at identifying challenges that occur when setting up studies and suggests solution strategies to address them. Three different institutes combining their experience discussed several studies in a workshop. Resulting main challenges are to find a suitable task, to operationalise the variables and to deal with a high analysis effort per participant. Automation in data evaluation and a detailed practical guideline on studies in design research are considered necessary.
\end{abstract}

Keywords: research methodologies and methods, design methods, engineering design, empirical study design, concept map

\section{Introduction}

Empirical studies form an important foundation stone in design research. On the one hand, the procedures of designers are examined descriptively. For example, the influence of experience with students and experienced designers is investigated. On the other hand, prescriptive methods are evaluated. The development and validation of design methods is a particularly important process. This validation should ensure - among others - that the methods have the desired effect in industry. However, many methods are not used in industry (Daalhuizen and Badke-Schaub, 2011). There are various reasons for an inconsistent use of methods in industrial context, although their use can provide benefits (Eilmus et al., 2012). Besides the missing link (Wallace, 2011) between academia and industry for an efficient transfer of methods, the reasons for the lack of use of many methods can often be traced back to a lack of method validation. Some methods are not put into practice, because they are described poorly (Birkhofer et al., 2002). Also, a low level of usability of the methods is cited as a reason (Jänsch, 2007). These deficits of design methods can be recognized and considered in empirical studies. The evaluation and validation of design methods in empirical studies would thus form a major contribution to the dissemination of design methods.

However, there are currently multiple aspects in the implementation of empirical studies in design research, which are not sufficiently considered. Dinar et al. (2015) have conducted a review of empirical studies in design and have identified various shortcomings in current studies. Current studies use mainly students as participants while working on constructed design problems. This raises the problem of a valid environment as students are not engineers yet and constructed tasks are only able to represent real 
engineering design problems partially. In most of the studies, a small number of participants are used (Dinar et al., 2015), what obstructs the acquisition of the necessary amount of data to make statistical analysis possible. Additionally, the data is often analysed manually and without a standardised framework. Most researchers use ad hoc categories, which are chosen by the research group. This makes a comparison of gained results very difficult (Dinar et al., 2015). Moreover, the fragmentation of the research community and a lack of a common research methodology are also cited as reasons why the research community does not achieve its full potential (Wallace, 2011).

There is currently a lack of suitable support for conducting valid empirical studies. There are overarching approaches for this, such as "Design Research Methodology" (DRM) according to Blessing and Chakrabarti (2009), which support the planning of design method development and validation. However, there is a lack of specific support for method researchers in the evaluation and validation of newly developed design methods (Marxen and Albers, 2012). The lack of clarity as to which methods and evidence are necessary for carrying out a successful validation continues to be a research gap in DRM (Gericke et al., 2017). The wide-ranging and rather holistic requirements for a study regarding objectivity (Cash et al., 2016), reliability and validity (Ruckpaul et al., 2014) as well as the systematic structure of the studies (Dinar et al., 2015) are often described. However, since the studies are primarily conducted by inexperienced researchers (Wallace, 2011), they lack experience in design practice and therefore are not fully able to implement real design problems in method validation. Although there are isolated approaches to support design researchers in setting up studies (e.g. the approach of Patel et al. (2019) on evaluating design prompts), they tend to focus on individual aspects of a study and are not widespread.

In the state of research, the requirements regarding objectivity, reliability and validity are clearly described. There are also approaches that provide support at a high level in the study design and implementation. Nevertheless, challenges and uncertainties frequently arise in the practical implementation of empirical studies in design research.

This contribution aims to draw attention to the challenges in empirical engineering design research and to describe first solution approaches based on an exchange of different researchers. This should create a certain awareness amongst young method developers with regards to valid design method validation and provide them with some inspiration on what to consider when setting up empirical research studies. We therefore conclude on the following research questions:

\section{Q1: What are current challenges in empirical engineering design research? \\ Q2: What are possible approaches to address those challenges?}

\section{Workshop-based approach to identify challenges and best practices}

In order to collect challenges and possible solution approaches in empirical engineering design research, a workshop was conducted. The workshop's aims, participants and structure are described in this section. The reason why a workshop was chosen can be traced to following rationale

i) allow for mutual inspiration and reveal synergies in between the workshop participants,

ii) being able to point out ambiguity in descriptions and aims and refine them on the spot,

iii) efficiency of communication and ideation.

\subsection{Workshop aims}

The main aim of the workshop was to share the individually accumulated knowledge as well as experience of the three institutions, find synergies and thus initialize a co-operational exchange of experience in empirical research in the field of engineering design. As described above, empirical studies are used in design research to investigate descriptive design approaches and to evaluate prescriptive design methods. The workshop focused on the evaluation and validation of design methods. However, the findings can also be applied to purely descriptive studies. The workshop participants exchanged examples of successful studies, failures as well as issues to consider both ways. Especially from these failures, participants wanted to learn and improve their own research. The presented studies were then 
used as basis to derive challenges as well as examples of best practices when conducting studies in empirical design research. Building up a common understanding of the topic should furthermore help to build up a continuous exchange of ideas and study designs.

\subsection{Team composition}

Six researchers from three different institutes participated in the workshop. The expertise of these researchers ranges from conducting experimental studies with student participants to studies with experienced designers in an industrial context. Their experience covers different types of studies from single case studies over interview studies to experiments, which implies descriptive as well as prescriptive designs. The researchers can demonstrate experience in experiments, case studies, surveys and interviews. The focus of research spans the development, evaluation and validation of different design methods. Various data collection methods, such as observation, protocol analysis, eye tracking and surveys among others have been used by the workshop's participants. Combined, the workshop participants observed about 220 participants in eye tracking studies, about 275 in experimental studies, about 530 participants in studies using classical methods - like survey, focus group and observation and over 50 participants in workshop environments.

\subsection{Workshop procedure}

The workshop took place in one of the participating institutes. After a round of introductions comprising the researchers and their corresponding expertise, the three institutes presented the studies they had carried out. The potential of sharing different study experiences and finding synergies together is also accompanied by certain challenges, such as making the studies comparable in various elements. Thus, a more standardized procedure to talk about the most important facts in these extensive studies has turned out to be necessary. In order to better compare the studies, the group decided to work with a published concept map on design method experiments, which was presented to the Design Society for feedback on the 30th Design for X Symposium 2019 (Üreten et al., 2019). Another way of dealing with the comparability of studies would have been a redefined structure of the presentations.

The concept map can be found in Figure 1. It is a visual depiction of the most important elements, which need to be considered when validating a design method through an experiment with participants. It mainly targets design researchers with little or no experience in conducting experiments with methods of empirical research in social sciences. The focus question addressed is "Which elements should be considered in an experimental study design to test design methods?". The elements are grouped in clusters and are connected to each other. For example, the experiment design is directly connected to data collection (see Figure 1), as data collection is a crucial part to think of when setting up an experiment design.

One cluster refers to the focus of the design method investigation and includes all elements specific to testing design methods in an experiment, such as validating single elements of a new method or validating an existing method in its full completeness (see Figure 1). This was used to discuss the presented studies' goals during the workshop. Another cluster refers to the basics of experimental design, which enabled the group to address the depicted different dimensions (like task, location, conditions and participants) in the discussion.

To derive solution approaches, it was necessary to define quality criteria and in which way those criteria can be achieved. Those criteria are also part of the concept map as objectivity, reliability and internal as well as external validity (see Figure 1). Those must be achieved by an appropriate choice of variables and a suitable operationalisation to make a valid measurement of those variables.

By using the concept map on design method experiments, a common language should be enhanced, a visual representation of the abstract presentations given as orientation and a consistent structure to compare the presented studies in between each other was facilitated. The focus of the presentations and the discussions were on the following four points.

1. Explaining the study, including its context, with strong orientation on the elements of the concept map, such as goal and hypothesis (see Figure 1) of the respective study.

2. The roadmap followed by each researcher - from developing a research question to publishing the study results in a scientific paper. 
3. Sharing and discussing the study implementation and occurring challenges - from the conception of study design to data analysis and interpretation.

4. Searching for solution strategies together with all participants in the workshop.

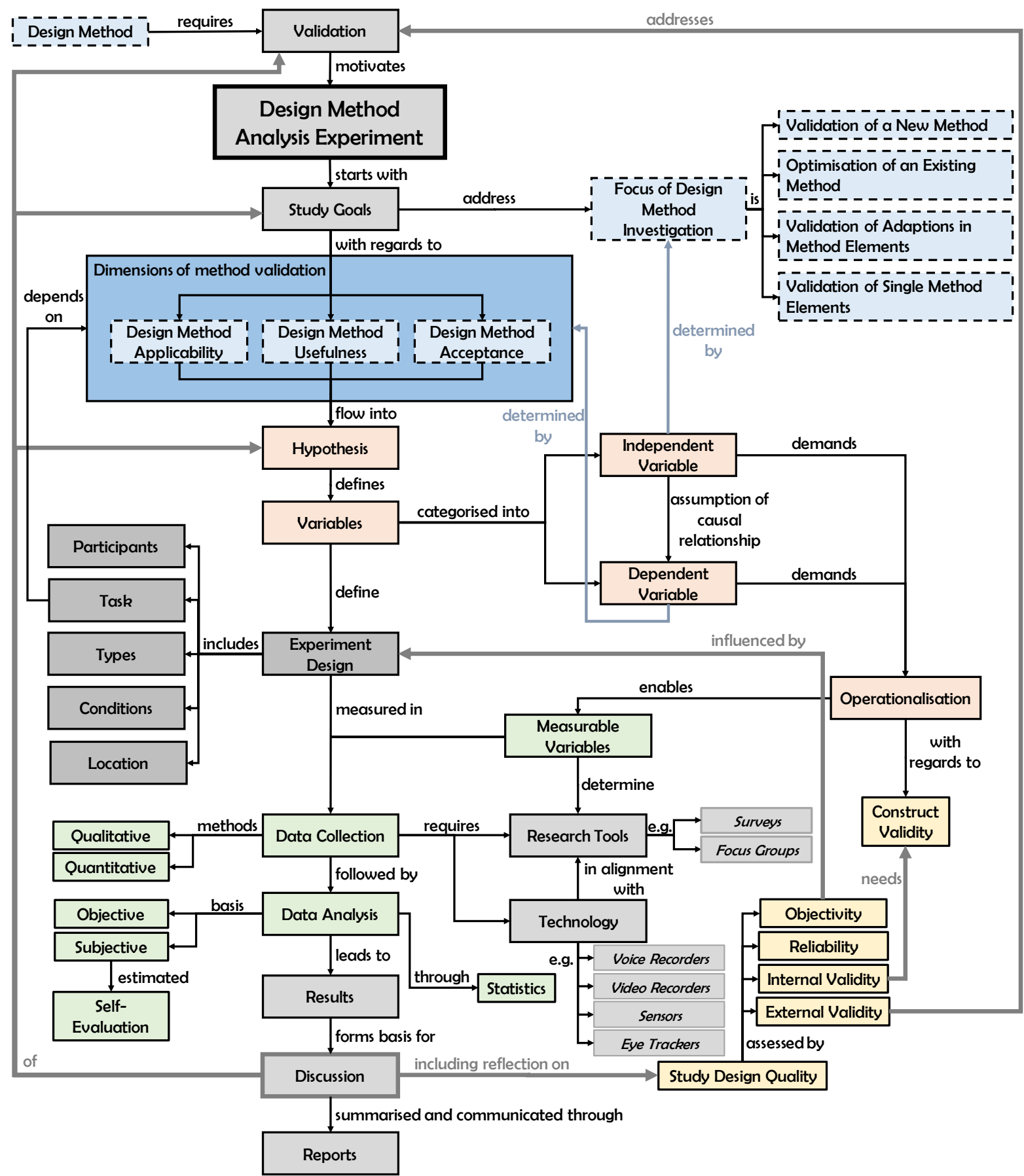

Figure 1. Concept map for design method experiments (Üreten et al. 2019)

In this part, the respective challenges and ambiguities of the individual institutes encountered were presented, discussed, generalised and clustered with the help of the visualisation in the concept map. Throughout the entire workshop a whiteboard was used for transparent documentation. From these ideas the challenges and best practises were derived. The concept map thus served as a connecting element between individual experiences made in the institutions and generalizable potential for further research in future, as best practices were incorporated into the concept map. All findings during the discussion were written down and clustered later. 


\section{Cases discussed}

In the following, the studies discussed in the workshop will be presented. They illustrate the broad range of expertise, the challenges derived and the best practices. Besides the studies' general structure, their intricacies as well as specific challenges were presented. The participants exchanged examples of successful studies, challenges as well as issues to consider both ways. Especially from these challenges, participants wanted to learn and improve their own research. Thus, the individual learnings in each institution were consolidated to a common pool of experiences in empirical design research through this workshop.

\subsection{Descriptive and prescriptive studies on the analysis of technical systems in engineering design}

Within the research project, a method was developed on the basis of successful approaches which supports the analysis of technical systems. First, a study was carried out to identify difficulties during the analysis of technical systems in engineering design (Matthiesen and Nelius, 2018b). Based on the findings, a method was adapted and evaluated in another study (Nelius and Matthiesen, 2019). Both studies were conducted using the same task, data collection methods and data analysis. A total of 34 participants - students and design engineers - worked alone on a realistic task from industry. Concurrent think aloud was used during the task to collect data. The procedure of the participants was recorded with a video camera and eye tracking glasses. To examine the confirmation bias, the participants' statements were transcribed and coded. The operationalisation of the confirmation bias in the statements in the concurrent think aloud proved to be difficult in the very open task. Therefore, a very detailed coding scheme had to be developed. The analysis of the observed approaches showed that students use the method more intensively than design engineers.

Usefulness of the method was observed by the change in the amount of correctly answered questions on the design task. The subjectively perceived usefulness of the method was additionally stated by the participants on a questionnaire. Here there were differences between the observed usefulness and the subjective usefulness. Students benefited from the method and achieved better results in their analysis, while no improvement could be shown for the design engineers. The subjective usefulness of the method, however, was rated significantly higher by the design engineers than by the students.

In an earlier study (Matthiesen and Nelius, 2018a), in addition to the think aloud, the eye tracking recordings of 7 participants were evaluated in order to analyse the visual attention. This provided further quantitative data. The eye tracking evaluation effort, however, would have been too great for the 34 participants.

\subsection{Experimental studies for method validation during solution finding}

The focus of this institute's cases lies on testing method adaptions. Single steps of a design method, which are either in the development phase or have already been fully developed, are taken and adapted systematically. In an experimental setup, typically the non-adapted form and the adapted form of the method are tested. The adaptions are derived from the target group's needs and focused through surveys and expert experiences with the design method. The adapted method steps are tested in comparison with their classical, non-adapted version. In several cases, a method aid was an adaption developed and tested (Üreten et al., 2017).

The presented studies focus on the process of solution finding. The tasks in these experiments have an open solution space. Experience with the procedure of working with engineering design catalogues according to Roth (1994) was collected in studies examining different adaptions in the procedure of using the catalogues (Üreten and Krause, 2017). Tasks ranged from theoretical tasks to real-application tasks from industrial context. In those studies, student participants solved tasks in workshops with group work as well as with individual work. The documentation was mainly accomplished through surveys and focus group interviews right after the study. Audio recording and observations during the study enriched the data basis. The solution approaches including the methodical procedure for solution finding were also presented and evaluated after the solution finding phase for measuring applicability, usefulness and acceptance. While the solutions of group and individual work have been collected, the focus has been on qualitative data concerning their process to solution finding. The evaluation consisted of a set of criteria 
concerning applicability, usefulness and especially acceptance of the adaption. The experiments yielded positive results - it was possible to identify boundary conditions under which method aids show success.

\subsection{Study on design method usefulness concerning manufacturability}

In this study, the aim was to investigate how design methods influence the design solution of engineers. It was investigated how two different design methods help students in the design for additive manufacturing. The work process of the participants was only observed qualitatively, not quantitatively. For this purpose, the leader of the study took notes on the different approaches and a qualitative survey was handed out after the study, asking participants on their opinion on the methods. The focus of the study was on the design solutions, not on the process. Therefore, only the design solutions are documented and evaluated quantitatively. In order to evaluate the design solutions they were graded in eight different categories, regarding additive manufacturing, summarized in a 16-point-scale by two judges.

In the study, the participants were first given an introduction to the field of additive manufacturing. With this knowledge the 30 students were invited to solve two design tasks. The resulting design was to be additively manufactured. By evaluation of the first task, the abilities of the participants to design for additive manufacturing can be deduced. Before working on the second task, the participants received an introduction to a method. In order to investigate two methods, the participants were divided into three subgroups. One group did not receive a further training and was used for comparison. The other two groups each received introductions to different design methods.

The quality of the participants' design solutions, determined in the second task, made it possible to assess the quality of the support. In order to determine the relative difficulty of the tasks, the participants are divided into two groups and work on the tasks in different order.

The advantage of this study design is that statistical measurable improvements can be found by calculating and including the different difficulties of the two tasks. The changed order of the task completion allows this calculation. Therefore, the internal validity of the results is relatively high.

The challenge remains in finding and acquiring enough participants to provide a reliable statistical data set. Since the solving of the design tasks is time intensive and participants need to stay focused, external motivation is needed in addition to internal motivation.

\section{Challenges and best practices discovered through the workshop}

The challenges of each study during its planning, conduction and analysis were presented systematically and discussed altogether. They can be clustered into different types and through the broad experience of the workshop participants, it was possible to have an engaged discussion with various solution approaches for the challenges. Best practices were noted and are documented in Table 1.

\section{Table 1. Summary of identified challenges and best practices}

\section{Challenges
Development of a valid operationalisation that can be implemented with available resources \\ Setting a solvable and challenging task that triggers the desired behavior \\ Uncertainty about the necessary number and qualifications of participants}

High effort and resources required for study and analysis

\section{Best Practices}

Conduct a pre-study to evaluate the chosen operationalisation

Use of complimentary data collection methods

Training of research staff for consistent data collection

Compare with other research fields for inspiration and standardised measures

Assess inter coder reliability

Conduct a pre-study to evaluate the suitability of the task

Ensure that the task provokes challenges that fit the design method

Choice of application example (product) - balancing daily/complex example of use

Correctness of design method usage should be evaluated or ensured

Acquisition of sufficient number of participants for statistical reliability

Check pre-knowledge in advance to have homogeneous groups

Choice of student participants or design engineers from industry depending on hypothesis

Evaluate smaller steps, adjust participant number

Automated task processing through proper operationalisation 


\subsection{Challenges}

The exchange revealed four major areas of challenges. The first one refers to the operationalisation. In a study, this would refer to the operationalisation of the latent and observable variable(s). The second issue refers to the adequate design of a task that is given in such an empirical study. The third concern is related to the study participants. This relates heavily with the fourth area of challenge, namely the effort and resources required to collect and analyse the data.

The operationalisation describes how the latent variable (e.g. creativity) is made measurable into an observable variable (e.g. number of generated ideas). The operationalisation of the independent and dependent variable in an empirical study is still an issue in engineering design. The cluster in the concept map, which shows this element (see Figure 1) is seen as a crucial point when setting up studies, as it has a major impact on the quality of the results that can be accomplished as well as the necessary effort and resources. As the proper definition of observable variables is key to an objective data collection, they need to be defined correctly and consistently.

When validating design methods, the operationalisation of the method's usefulness is an important step. Due to the many different purposes of methods, however, there is no uniform operationalisation of method usefulness. In other academic disciplines such standardised operationalisations exist. Intelligence for example is typically measured with an IQ test, which results in an IQ value for the participant. The way of measuring and also having standardised measurements for certain variables, such as system understanding for analysis methods or creativity for synthesis methods remain unclear, non-uniform and need to be researched for further studies.

Also, the operationalisation of design approaches is often vague and not uniform. Especially for design approaches there is a close relationship between operationalisation and data collection method. The concurrent think aloud method for instance may help to understand the participants' thoughts, if applied correctly. Some studies presented in the workshop explained the crucial role of study observers - as every observation is subjective to a certain degree - and their a priori training. Also, the trainers need a mutual understanding if they train observers for the same data collection during a study. The same is valid for coders, who analyse the data after the study as they may influence the results by subjective interpretation. When analysing the collected data, a manual coding of the data is usually necessary. The reliability of the operationalisation can be evaluated by a double coding and the determination of the inter coder reliability. For the double coding, a very large expenditure of time is necessary. As long as there are no standardised operationalisations, this effort is driven by many researchers in parallel without them profiting from each other. This also leads to the problem that results cannot be compared through different operationalisations.

The task or design prompt that is given in an empirical study to be solved by the participants can be found in the cluster for the experiment design in the concept map (see Figure 1). Challenges arise in measuring the difficulty of a task. Especially in in-between subject designs with two subsequent tasks of required equal difficulty, the assurance of the level of the task needs to be cleared.

Furthermore, it is a challenge to reconstruct a task from industry into a task within an academic, laboratory environment because the participants lack the necessary background knowledge. It is often unclear how much input is required to depict the required context and when this input gives too much information, which may influence the participants' behaviour in an undesired way. In empirical engineering design studies the development of a technical system is often the core of the task. This raises the question about what kind of product, device or material, should be provided to the participants during a study. Finding a balance between constructed design tasks on daily products (e.g. coffee machine) and realistic design tasks on specialised systems is not necessarily straightforward and requires a great deal of experience. Combining the challenges of designing a proper task and using a specific illustrative product, it still does not guarantee that the task triggers the assumed or required behaviour connected with the method or issue that is investigated.

Another issue is the objective evaluation of the solutions developed by the study participants. While evaluation can take place through experts, open solution tasks make a clear assessment more challenging and certain measures need to be taken to reduce that effect. There is a lack of a guideline to define a design task, which evokes realistic challenges that can be addressed within a reasonable timeframe and where depended variables can be measured by the chosen operationalisation. 
With regards to the participants there are a lot of criteria for including and excluding participants (see Figure 1). Especially their knowledge needs to be considered for empirical studies. Also, for gaining statistically reliable data, the number of participants with the required qualifications, such as experience and motivation, should be adequate. Creating a group consisting of sufficiently experienced engineers with homogeneous experience and training is thus a great deal of challenge. Especially because experiential knowledge is usually connected to a certain product. This connects to the task as the system of choice might be unknown by some participants. The type of data collection can also impose requirements on the participants. With concurrent think aloud, some participants experience difficulties in not being able to verbalise their thoughts fluently. When eye tracking is used, subjects with glasses are frequently excluded from the study.

If the challenge of having a sufficient number of participants for a statistically reliable dataset is met, this often results in high effort and resources required for data analysis. Especially qualitative data can be quite rigorous to evaluate. Transcripts for coding of sessions, double resources for determining the inter coder reliability and personnel required during the conduction of the empirical research, such as having enough study observers and training them beforehand, heavily relies on the resources available and poses limitations. The effort required to carry out and analyse the study is primarily associated with the chosen operationalisation and data collection method.

\subsection{Best practices}

The best practices are derived based on the challenges encountered and the discussions made with all participants. Regarding the issues with operationalisation, a pre-study is strongly recommended to evaluate and test the operationalisation and data collection method. Though in general a pre-study is recommended to have a look at each instance and element of a rigorous planning, especially the operationalisation and the task including the uncertainty about the possible behaviour is to be evaluated. To encounter the challenges arising from the use of a single data collection method, methods can be combined. For example, through triangulation, additional information can be generated to ensure more valid conclusions by comparing different data sets. This is also accompanied by a raised effort for the additional methods employed.

Our experience is, that there is a trend to go into more detail and to use quantifiable data. Though there is no standardised way of doing research, the methods and data collection tools used should be considered carefully with the issue that needs to be addressed or investigated. Also, the number of participants and the target audience plays an important role. During the design method use, it should be foreseen that the method is applied correctly, instead of realising after the study that the method was not applied in a way, which was required for the study. It has been effective to not only test the condition "no method" and "method application", but also to compare similar design methods or method adaptions. As a lot of valuable insights can be gained with empirical studies, it is recommended to start considering an empirical study framework or context (such as study design, task, evaluation) for method validation.

Automation in data analysis enables the statistical evaluation of large numbers of participants at a reasonable effort. Concerning this, it has to be considered that the key to automation is a proper operationalisation of latent variables that lead to variables that can be measured. By using algorithms, those variables can be extracted from the extensive amount of data necessary for statistical analysis. These algorithms only produce valid results when they are created by using valid operationalisations. The efficient data analysis using algorithms promotes the dissemination of standardised operationalisations and thus contributes to comparable results.

\section{Reflection about the workshop approach and findings}

As intended, the workshop approach enabled to prevent ambiguities and misunderstandings by face to face discussion. The used concept map additionally supported in gaining a common understanding. Researchers participating in the workshop represent three different research poles with different types of empirical studies (experiments, surveys, interviews, case studies) as well as the use of different resources such as expert interviews, design methods used, data collection methods, types of evaluation and operational conduction. 
This work bears certain limitations. Firstly, the limited number of researchers and the cases discussed resulting from own research work of the researchers' institutions. The participants may have been biased depending solely on the limited amount of studies discussed. Secondly, the researchers involved are all from European countries, which leaves out possible views from other design communities. Regarding these limitations, the results are a first step for raising awareness for some challenges in design research faced by young researchers and are therefore not be seen as an exhaustive set of challenges. Extensions to this initial work as a kick-off can be made for further detailing the work

a) in the approach to answer the research questions by enriching the research experiences through other, complimentary research methods. Future work should include a detailed analysis of existing cases, such as a systematic literature review and in case of workshop, increase the number of participants with regards to generalisability of the findings,

b) by aligning individual best practices into a line of thought or flow of preparation for empirical studies and c) with a survey that could validate the findings and increase representativeness.

\section{Conclusion and outlook}

The requirements for valid studies in design research are high and extensive. Especially for the mostly inexperienced researchers who set up and execute the studies, considerable challenges arise. The challenges can be assigned to two overarching aspects. (1.) The systematic procedure of preparing, building up a design task, carrying out and evaluating a study. The approaches described in the literature describe the procedures too abstractly and provide too little support in practical implementation. A detailed guideline for studies in design research for inexperienced researchers is not available. (2.) The operationalisation of the target variables represents a major difficulty, resulting in a high effort. The suitable operationalisation represents a basic requirement for every data collection. However, there are no standardised research methods, which support operationalisation in this area so far. In addition, the validation effort of today's research methods, thus the available resources, reduces the possible number of participants and the number of studies. The findings have been based on a workshop with 6 researchers from different universities representing various experiences, and need to be further examined by detailing the findings and complimentary research to consider generalisability of the findings. With this contribution we would like to stimulate a discussion in the research community in order to work together on these research challenges. Both a written guideline for the development of empirical studies as well as standardised (and possibly automated) research methods could form a major contribution to design research.

\section{Acknowledgements}

We would like to thank all studies' participants, data collectors and analysers, who enabled a data basis rich enough to be analysed systematically and thus contributed to empirical design research in engineering design. We wish to thank all who gave us valuable feedback for the concept map: thanks for providing us with insights and experience with which we are able to improve the concept map.

\section{References}

Birkhofer, H. et al. (2002), "Why Methods Don't Work and How to Get them to Work", Engineering design in integrated product development: Design methods that work; Proceedings of the 3rd international seminar and workshop held in University of Zielona Góra, 10th-12th October 2002, Zielona Góra, Poland, R. Rohatyński, Zielona Góra, pp. 29-36.

Blessing, L.T.M. and Chakrabarti, A. (2009), DRM, a Design Research Methodology, Springer London, London. https://doi.org/10.1007/978-1-84882-587-1

Cash, P., Stanković, T. and Štorga, M. (2016), "An introduction to experimental design research”, In: Cash, P., Stanković, T., Štorga, M. (Eds.), Experimental Design Research, Springer, Cham, pp. 3-12. https://doi.org/ 10.1007/978-3-319-33781-4_1

Daalhuizen, J. and Badke-Schaub, P. (2011), "The use of methods by advanced beginner and expert industrial designers in non-routine situations: a quasi-experiment”, International Journal of Product Development, Vol. 15 No. 1/2/3, pp. 54 -70. https://doi.org/10.1504/IJPD.2011.043661 
Dinar, M. et al. (2015), "Empirical studies of Designer Thinking: Past, Present, and Future", Journal of Mechanical Design, Vol. 137 No. 2, pp. 21101.1-13. https://doi.org/10.1115/1.4029025

Eilmus, S. et al. (2012), "Evaluating a methodical approach for developing modular product families in industrial case studies", DS 70: Proceedings of DESIGN 2012, the 12th International Design Conference, Dubrovnik, Croatia, The Design Society, Glasgow, pp. 837-846.

Gericke, K., Eckert, C. and Stacey, M. (2017), "What do we need to say about a design method?", DS 87-7 Proceedings of the 21st International Conference on Engineering Design (ICED 17) Vol 7: Design Theory and Research Methodology, 21-25 August 2017, Vancouver, Canada, The Design Society, Glasgow, pp. 101-110.

Jänsch, J. (2007), Akzeptanz und Anwendung von Konstruktionsmethoden im industriellen Einsatz: Analyse und Empfehlungen aus kognitionswissenschaftlicher Sicht [PhD Thesis], Produktentwicklung und Maschinen elemente, Technical University of Darmstadt.

Marxen, L. and Albers, A. (2012), "Supporting Validation in the Development of Design Methods", DS 70: Proceedings of DESIGN 2012, the 12th International Design Conference, Dubrovnik, Croatia, The Design Society, Glasgow, pp. 1009-1018.

Matthiesen, S. and Nelius, T. (2018a), "Eye tracking study on successful micro-strategies by design engineers for the synthesis-driven analysis of technical systems", Proceedings of TMCE 2018, 7-11 May, 2018, Las Palmas de Gran Canaria, Spain, University of Technology, Delft, pp. 295-304.

Matthiesen, S. and Nelius, T. (2018b), "Managing Assumptions during Analysis-Study on successful Approaches of Design Engineers", DS 91: Proceedings of NordDesign 2018, 14-17 August 2018, Linköping, Sweden, The Design Society, Glasgow.

Nelius, T. and Matthiesen, S. (2019), "Experimental Evaluation of a Debiasing Method for Analysis in Engineering Design", Proceedings of the 22nd International Conference on Engineering Design (ICED19), Cambridge University Press, pp. 489-498. https://doi.org/10.1017/dsi.2019.53

Patel, A., Elena, M.-V. and Summers, J. (2019), "A Systematic Approach to Evaluating Design Prompts in Supporting Experimental Design Research", Proceedings of the 22nd International Conference on Engineering Design (ICED19), Cambridge University Press, pp. 2755-2764. https://doi.org/10.1017/ dsi.2019.282

Roth, K. (1994), Konstruieren mit Konstruktionskatalogen, Springer-Verlag Berlin Heidelberg, New York. https://doi.org/10.1007/978-3-642-17467-4

Ruckpaul, A., Kriltz, A. and Matthiesen, S. (2014), "Using Eye Tracking to Understand the Engineering Designers' Behaviour in Synthesis Driven Analyzing Processes - Experiences in Study Design", Analyzing Cognitive Processes During Design: Proceedings of the HBiD 2014, ETH Zurich, Product Development Group, Zurich.

Üreten, S. et al. (2017), "Continuing Education and Personalization of Design Methods to Improve their Acceptance in Practice - An Explorative Study”, Procedia CIRP, Vol. 60, pp. 524-529. https://doi.org/ 10.1016/j.procir.2017.01.012

Üreten, S. et al. (2019), “A Concept Map for Design Method Experiments in Product Development - A Guideline for Method Developers", DS 98: Proceedings of the 30th Symposium Design for X (DFX 2019), 18-19 September 2019, Jesteburg, Germany. https://doi.org/10.35199/dfx2019.13

Üreten, S. and Krause, D. (2017), "Discursive vs. intuitive - An experimental study to facilitate the use of design catalogues", DS 87-7 Proceedings of the 21st International Conference on Engineering Design (ICED 17) Vol 7: Design Theory and Research Methodology, 21-25 August 2017, Vancouver, Canada, The Design Society, Glasgow, pp. 99-108.

Wallace, K. (2011), “Transferring Design Methods into Practice”, In: Birkhofer, H. (Ed.), The Future of Design Methodology, Springer, London, pp. 239-248. 